\title{
CLASSICAL SOLUTIONS OF LINEAR REGULATOR FOR DEGENERATE DIFFUSIONS
}

MD. AZIZUL BATEN

Received 26 November 2005; Revised 19 April 2006; Accepted 25 April 2006

We study a stochastic control problem for linear degenerate systems. We establish the existence of a classical solution of the degenerate Bellman equation by the technique of viscosity solutions, and the optimal policy is shown to exist from the optimality conditions.

Copyright (c) $2006 \mathrm{Md}$. Azizul Baten. This is an open access article distributed under the Creative Commons Attribution License, which permits unrestricted use, distribution, and reproduction in any medium, provided the original work is properly cited.

\section{Introduction}

The usual framework of control is the one given in probably the most studied control problem, stochastic regulator control problem, which deals with minimizing a performance index of a system governed by a set of differential equations. The stochastic linear regulator problem has been studied by many authors including Bensoussan [4], Fleming and Soner [9] for nondegenerate diffusions. Da Prato [8] gives the solution to the stochastic linear regulator for the degenerate systems related to Riccati equations (i.e., any ordinary differential equation) for the quadratic case with infinite horizon. But he has not established the existence of a classical solution of the Hamilton-Jacobi-Bellman (HJB) equation (i.e., a partial differential equation in the optimal control theory) to the linear regulator control problem. Here we have studied an extended stochastic control problem of the linear regulator for the degenerate diffusions by considering the general case with infinite horizon.

We are concerned with the stochastic control problem to minimize the discounted expected cost:

$$
J(c)=E\left[\int_{0}^{\infty} e^{-\alpha t}\left\{f\left(x_{t}\right)+\left|c_{t}\right|^{m}\right\} d t\right]
$$

Hindawi Publishing Corporation

Journal of Applied Mathematics and Stochastic Analysis

Volume 2006, Article ID 98764, Pages 1-16

DOI 10.1155/JAMSA/2006/98764 
2 Classical solutions of linear regulator for degenerate diffusions

over $c \in \mathscr{A}$ subject to the degenerate stochastic differential equation

$$
d x_{t}=\left[A x_{t}+c_{t}\right] d t+\sigma x_{t} d w_{t}, \quad x_{0}=x \in \mathbb{R}, t \geq 0
$$

for $\alpha>0, m \geq 0$, nonzero constants $A, \sigma \neq 0$, and a continuous function $f$ on $\mathbb{R}$ such that

$$
\begin{gathered}
0 \leq f(x) \leq K\left(1+|x|^{m}\right), \quad x \text { in } \mathbb{R}, \\
k_{0}|x|^{m}-k_{1} \leq f(x)
\end{gathered}
$$

for some constants $K, k_{0}, k_{1}>0$. Here, $\left(w_{t}\right)$ is a one-dimensinal standard Brownian motion on a complete probability space $\left(\Omega, \mathscr{F}_{F}, P\right)$ endowed with the natural filtration $\mathscr{F}_{t}$ generated by $\sigma\left(w_{s}, s \leq t\right)$, and $\mathscr{A}$ denotes the class of all $\mathscr{F}_{t}$-progressively measurable processes $c=\left(c_{t}\right)$ with $J(c)<\infty$.

We also refer to Bensoussan [4], Fleming and Soner [9] for nondegenerate diffusions, and also Da Prato [8] for the degenerate stochastic system from the view of Riccati equations in case of $f(x)=K x^{2}$ and $m=2$.

The main purpose of the linear regulator problem (1.1) and (1.2) is to give a synthesis of optimal control for degenerate stochastic systems by a classical solution $u$ of the associated HJB equation

$$
H\left(x, u, u^{\prime}, u^{\prime \prime}\right):=\alpha u-\left\{\frac{1}{2} \sigma^{2} x^{2} u^{\prime \prime}+A x u^{\prime}+\min _{r \in \mathbb{R}}\left(|r|^{m}+r u^{\prime}\right)+f(x)\right\}=0 \quad \text { in } \mathbb{R},
$$

where $\alpha>0, u_{x}, u_{x x}$ are partial derivatives of $u(x, t)$ with respect to $x$. Generaly speaking, the difficulty stems from the degeneracy in the second-order term of (1.5).

Our objective is to find the viscosity solution for $u$ of (1.5) following Bardi and Capuzzo-Dolcetta [2], Crandall et al. [6], Fleming and Soner [9] through the limit of the solution $v=v_{L}, L>0$, to the HJB equation

$$
\alpha v_{L}(r)-\left\{\frac{1}{2} \sigma^{2} x^{2} v_{L}^{\prime \prime}(r)+A x v_{L}^{\prime}(r)+\min _{|r| \leq L}\left(|r|^{m}+r v_{L}^{\prime}(r)\right)+f(x)\right\}=0 \quad \text { in } \mathbb{R}
$$

as $L \rightarrow \infty$, where the value function $v_{L}$ can be defined as a function whose value is the minimum value of the objective function of the control problem for the system, that is,

$$
v_{L}(t, x)=\inf _{c \in \mathscr{A}_{L}} J(c) \quad \text { as } L \longrightarrow \infty,
$$

where $\mathscr{A}_{L}=\left\{\right.$ for all $c=\left(c_{t}\right) \in \mathscr{A}$ such that $\left|c_{t}\right| \leq L$ for all $\left.t \geq 0\right\}$. Also our study deals with the smoothness of the viscosity solution $u$ of (1.5) using a convexity argument of $v_{L}(x)$ and $u(x)$.

To this end, we assume that $f$ is uniformly continuous with $m$-weight, that is, there exists $\mathbf{C}_{\rho}>0$, for any $\rho>0$, such that

$$
|f(x)-f(y)| \leq C_{\rho}|x-y|^{m}+\rho\left(1+|x|^{m}+|y|^{m}\right) \quad \forall x, y \in \mathbb{R} .
$$

We notice that (1.8) is fulfilled for $f(x)=|x|^{\mu}, 0 \leq \mu \leq m$, and plays an important role as treated in Koike and Morimoto [12], Menaldi and Robin [14]. 
In Section 2, we show that the value function $u(x):=\lim _{L \rightarrow \infty} v_{L}(x)$ is a viscosity solution of (1.5). Section 3 is devoted to the study of smoothness of $u$. Finally, in Section 4, we present an optimal control of the control problem (1.1) and (1.2).

\section{Viscosity solutions}

We studied here the properties of the value function $v_{L}(x)$ using the method of dynamic programming, initiated by Bellman [3] and and showed that $v_{L}(x)$ converges to a viscosity solution $u(x)$ of the Bellman equation (1.5).

The notion of viscosity solutions to HJB equation was introduced by Crandall and Lions [7] in the early 80's and requires only the solution to be continuous and by Lions [13] for second-order equations. Crandall et al. [6] showed a modern presentation of this notion of solution in the User's Guide to viscosity solutions and Fleming and Soner [9] also described the connections with control problems. Bardi and Capuzzo-Dolcetta [2] showed an introduction to the theory limited to first-order equations. They also characterized the value function as a viscosity solution of HJB equation.

Let $\omega: \mathbb{R} \mapsto \mathbb{R}$ be a scalar function, defined on an open set $\mathbb{R} \subseteq \mathbb{R}^{2}$. In the following, we consider the second-order, partial differential equation

$$
\mathbf{H}\left(\mathbf{x}, \boldsymbol{\omega}(\mathbf{x}), \boldsymbol{\omega}^{\prime}(\mathbf{x}), \boldsymbol{\omega}^{\prime \prime}(\mathbf{x})\right)=\mathbf{0} \text { in } \mathbb{R} .
$$

Here $H: \mathbb{R} \times \mathbb{R} \times \mathbb{R} \times \mathbb{R} \rightarrow \mathbb{R}$ is a continuous (nonlinear) function.

The set of superdifferentials of $\omega$ at a point $x$ is defined as

$$
J^{2,+} \omega(x)=\left\{(p, q) \in \mathbb{R}^{2}: \limsup _{y \rightarrow x} \frac{\omega(y)-\omega(x)-p \cdot(y-x)-(1 / 2) q|y-x|^{2}}{|y-x|^{2}} \leq 0\right\} .
$$

Similarly, the set of subdifferentials of $\omega$ at a point $x$ is defined as

$$
J^{2,-} \omega(x)=\left\{(p, q) \in \mathbb{R}^{2}: \liminf _{y \rightarrow x} \frac{\omega(y)-\omega(x)-p \cdot(y-x)-(1 / 2) q|y-x|^{2}}{|y-x|^{2}} \geq 0\right\},
$$

where $(\cdot)$ stands for the scalar product of two vectors in $\mathbb{R}^{2}$.

We recall by Crandall et al. [6] the definition of viscosity solutions of (2.1) in terms of sub- and superdifferentials.

Definition 2.1. A function $\omega \in C(\mathbb{R})$ is called a viscosity subsolution of (2.1) if,

$$
\mathbf{H}(\mathbf{x}, \boldsymbol{\omega}(\mathbf{x}), \mathbf{p}, \mathbf{q}) \leq \mathbf{0} \quad \text { for every } \mathbf{x} \in \mathbb{R} \forall(\mathbf{p}, \mathbf{q}) \in \mathbf{J}^{2,+} \boldsymbol{\omega}(\mathbf{x}) .
$$

Similarly, a function $\omega \in C(\mathbb{R})$ is called a viscosity supersolution of $(2.1)$ if

$$
\mathbf{H}(\mathbf{x}, \boldsymbol{\omega}(\mathbf{x}), \mathbf{p}, \mathbf{q}) \geq \mathbf{0} \quad \text { for every } \mathbf{x} \in \mathbb{R} \forall(\mathbf{p}, \mathbf{q}) \in \mathbf{J}^{2,-} \boldsymbol{\omega}(\mathbf{x}) .
$$

$\omega \in C(\mathbb{R})$ is a viscosity solution of $(2.1)$ if it is both viscosity sub- and supersolutions of (2.1).

We now turn to the second definition of a viscosity solution which is equivalent to the previous one. 
4 Classical solutions of linear regulator for degenerate diffusions

Equivalent definition. A function $\omega \in C(\mathbb{R})$ is called a viscosity subsolution of (2.1) if, for every $\varphi \in C^{2}(\mathbb{R}), \omega-\varphi$ has a local maximum at $x \in \mathbb{R}$, then

$$
\mathbf{H}\left(\mathbf{x}, \boldsymbol{\omega}(\mathbf{x}), \varphi^{\prime}(\mathbf{x}), \varphi^{\prime \prime}(\mathbf{x})\right) \leq \mathbf{0} .
$$

Similarly, a function $\omega \in C(\mathbb{R})$ is called a viscosity supersolution of (2.1) if, for every $\varphi \in C^{2}(\mathbb{R}), \omega-\varphi$ has a local minimum at $x \in \mathbb{R}$, then

$$
\mathbf{H}\left(\mathbf{x}, \boldsymbol{\omega}(\mathbf{x}), \varphi^{\prime}(\mathbf{x}), \varphi^{\prime \prime}(\mathbf{x})\right) \geq \mathbf{0}
$$

In order to ensure the integrability of $J(c)$, we assume that

$$
-\alpha+\frac{1}{2} \sigma^{2} m(m-1)+m|A|<0 .
$$

Lemma 2.2. Let $2 \leq \mu \leq m$. Then, under (2.8),

$$
E\left[e^{-\alpha t}\left|x_{t}\right|^{\mu}+\int_{0}^{t} e^{-\alpha s}\left|x_{s}\right|^{\mu} d s\right] \leq \kappa_{\mu}\left(|x|^{\mu}+E\left[\int_{0}^{t} e^{-\alpha s}\left|c_{s}\right|^{\mu} d s\right]\right),
$$

for some constant $\kappa_{\mu}>0$, depending on $\mu$.

Proof. By (2.8), we take $\beta \in(0, \alpha)$ such that

$$
-\beta+\frac{1}{2} \sigma^{2} \mu(\mu-1)+\mu|A|<0 .
$$

Itô's formula gives

$$
\begin{aligned}
e^{-\alpha t}\left|x_{t}\right|^{\mu}=|x|^{\mu}+\int_{0}^{t} e^{-\alpha s}\{ & -\alpha\left|x_{s}\right|^{\mu}+\mu\left|x_{s}\right|^{\mu-1} \operatorname{sgn}\left(x_{s}\right)\left(A x_{s}+c_{s}\right) \\
& \left.+\frac{1}{2} \mu(\mu-1) \sigma^{2}\left|x_{s}\right|^{\mu}\right\} d s+\int_{0}^{t} e^{-\alpha s} \mu\left|x_{s}\right|^{\mu} \sigma d w_{s} \\
= & |x|^{\mu}+\int_{0}^{t} e^{-\alpha s}\left(-\alpha+\frac{1}{2} \mu(\mu-1) \sigma^{2}+\mu|A|+\beta-\beta\right)\left|x_{s}\right|^{\mu} d s \\
& +\int_{0}^{t} e^{-\alpha s} \mu\left|x_{s}\right|^{\mu-1} \operatorname{sgn}\left(x_{s}\right)\left|c_{s}\right| d s+\int_{0}^{t} e^{-\alpha s} \mu\left|x_{s}\right|^{\mu} \sigma d w_{s} .
\end{aligned}
$$

Using a usual localizing argument, we have by (2.10),

$$
\begin{aligned}
E\left[e^{-\alpha\left(\tau_{n} \wedge t\right)}\left|x_{\tau_{n} \wedge t}\right|^{\mu}\right] \leq & |x|^{\mu}+E\left[\int_{0}^{\tau_{n} \wedge t} e^{-\alpha s}\{-(\alpha-\beta)\}\left|x_{s}\right|^{\mu} d s\right] \\
& +E\left[\int_{0}^{\tau_{n} \wedge t} e^{-\alpha s} \mu\left|x_{s}\right|^{\mu-1} \operatorname{sgn}\left(x_{s}\right)\left|c_{s}\right| d s\right] .
\end{aligned}
$$


So

$$
\begin{aligned}
E\left[e^{-\alpha t}\left|x_{t}\right|^{\mu}+(\alpha-\beta) \int_{0}^{t} e^{-\alpha s}\left|x_{s}\right|^{\mu} d s\right] & \leq|x|^{\mu}+E\left[\int_{0}^{t} e^{-\alpha s} \mu\left|x_{s}\right|^{\mu-1} \operatorname{sgn}\left(x_{s}\right)\left|c_{s}\right| d s\right] \\
& \leq|x|^{\mu}+E\left[\int_{0}^{t} e^{-\alpha s} \mu\left|x_{s}\right|^{\mu-1}\left|c_{s}\right| d s\right] .
\end{aligned}
$$

We set $q=\mu /(\mu-1)$ and choose $\gamma>0$ such that $(\beta-\alpha) / 2+(1 / q) \gamma^{q} \mu^{q}<0$. Then, by Young's inequality,

$$
\begin{aligned}
\mu\left|x_{s}\right|^{\mu-1}\left|c_{s}\right| & \leq \frac{1}{q}\left(\gamma \mu\left|x_{s}\right|^{\mu-1}\right)^{q}+\frac{1}{\mu}\left(\frac{1}{\gamma}\left|c_{s}\right|\right)^{\mu} \\
& \leq \frac{\alpha-\beta}{2}\left|x_{s}\right|^{\mu}+\frac{1}{\mu \gamma^{\mu}}\left|c_{s}\right|^{\mu} .
\end{aligned}
$$

Therefore, we deduce (2.9).

Theorem 2.3. Assume (1.3), (1.4), (1.8), and (2.8). Then

$$
\begin{aligned}
& \qquad \leq v_{L}(x) \leq \bar{K}\left(1+|x|^{m}\right), \\
& v_{L}(x) \text { fulfills (1.8) with some constant } C_{\rho} \text {, independent of } L,
\end{aligned}
$$

where $\bar{K}=K\left(1 / \alpha+\kappa_{m}\right)$.

Proof. Let $\left(z_{t}\right)$ be the unique solution of

$$
d z_{t}=A z_{t} d t+\sigma z_{t} d w_{t}, \quad z_{0}=x
$$

Then, by (1.3) and Lemma 2.2,

$$
\begin{aligned}
v_{L}(x) \leq E\left[\int_{0}^{\infty} e^{-\alpha t} f\left(z_{t}\right) d t\right] & \leq K E\left[\int_{0}^{\infty} e^{-\alpha t}\left(1+\left|z_{t}\right|^{m}\right) d t\right] \\
& \leq \frac{K}{\alpha}+K \kappa_{m}\left(|x|^{m}+E\left[\int_{0}^{\infty} e^{-\alpha t}\left|c_{t}\right|^{m} d t\right]\right) \\
& =\frac{K}{\alpha}+K \kappa_{m}|x|^{m} \\
& \leq \bar{K}\left(1+|x|^{m}\right),
\end{aligned}
$$

which implies (2.15). To prove (2.16), we set $J_{c}(x)=J(c)$ and denote by $C_{L}$ the class of all $c \in \mathscr{A}_{L}$ satisfying

$$
E\left[\int_{0}^{\infty} e^{-\alpha t}\left|x_{t}\right|^{m} d t\right] \leq \theta\left(1+|x|^{m}\right)
$$


6 Classical solutions of linear regulator for degenerate diffusions

where $\theta=\left(\bar{K}+1+k_{1} / \alpha\right) / k_{0}$. Since there exists $c \in \mathscr{A}_{L}$, for any integer $n \geq 1$, such that $J_{c}(x) \leq v_{L}(x)+1 / n$, we have by (1.4) and (2.15),

$$
k_{0} E\left[\int_{0}^{\infty} e^{-\alpha t}\left|x_{t}\right|^{m} d t\right]-\frac{k_{1}}{\alpha} \leq\left(\bar{k}+\frac{1}{n}\right)\left(1+|x|^{m}\right) .
$$

This yields that such $c$ belongs to $C_{L}$, and thus

$$
v_{L}(x)=\inf _{c \in \mathscr{C}_{L}} J_{c}(x)
$$

Let $\left(y_{t}\right)$ be the solution $y_{t}$ of (1.2) with $y_{0}=y$. It is clear that $x_{t}-y_{t}$ fulfills (2.17) with initial condition $x-y$. Hence, by (1.8), (2.21), and Lemma 2.2,

$$
\begin{aligned}
\left|v_{L}(x)-v_{L}(y)\right| & \leq \sup _{c \in \mathscr{C}_{L}}\left|J_{c}(x)-J_{c}(y)\right| \\
& \leq \sup _{c \in \mathscr{C}_{L}} E\left[\int_{0}^{\infty} e^{-\alpha t}\left|f\left(x_{t}\right)-f\left(y_{t}\right)\right| d t\right] \\
& \leq \sup _{c \in \mathscr{C}_{L}} E\left[\int_{0}^{\infty} e^{-\alpha t}\left\{C_{\bar{\rho}}\left|x_{t}-y_{t}\right|^{m}+\bar{\rho}\left(1+\left|x_{t}\right|^{m}+\left|y_{t}\right|^{m}\right)\right\} d t\right] \\
& \leq C_{\bar{\rho} \kappa_{m}}|x-y|^{m}+\frac{\bar{\rho}}{\alpha}+2 \bar{\rho} \theta\left(1+|x|^{m}+|y|^{m}\right) \\
& =C_{\rho}|x-y|^{m}+\rho\left(1+|x|^{m}+|y|^{m}\right),
\end{aligned}
$$

where $C_{\rho}=C_{\bar{\rho}} \kappa_{m}$ and $\bar{\rho}=\rho /(1 / \alpha+2 \theta)$. Therefore, we deduce (2.16), completing the proof.

THEOREM 2.4. Under the assumptions of Theorem 2.3, the dynamic programming principle holds for $v_{L}(x)$, that is, for any stopping time $\tau$,

$$
v_{L}(x)=\inf _{c \in A_{L}} E\left[\int_{0}^{\tau} e^{-\alpha t}\left\{f\left(x_{t}\right)+\left|c_{t}\right|^{m}\right\} d t+e^{-\alpha \tau} v_{L}\left(x_{\tau}\right)\right]
$$

Proof. We denote $\bar{v}(x)$ the right-hand side of (2.23). By the formal Markov property,

$$
E\left[\int_{\tau}^{\infty} e^{-\alpha t}\left\{f\left(x_{t}\right)+\left|c_{t}\right|^{m}\right\} d t \mid \mathscr{F}_{\tau}\right]=e^{-\alpha \tau} J_{\widetilde{c}}\left(x_{\tau}\right),
$$

with $\tilde{c}$ equal to $c$ shifted by $\tau$. Thus

$$
\begin{aligned}
J_{c}(x) & =E\left[\int_{0}^{\tau}+\int_{\tau}^{\infty} e^{-\alpha t}\left\{f\left(x_{t}\right)+\left|c_{t}\right|^{m}\right\} d t\right] \\
& \geq E\left[\int_{0}^{\tau} e^{-\alpha t}\left\{f\left(x_{t}\right)+\left|c_{t}\right|^{m}\right\} d t+e^{-\alpha \tau} v_{L}\left(x_{\tau}\right)\right] .
\end{aligned}
$$


It is known in Fleming and Soner [9], Nisio [15] that this formal argument can be verified, and we deduce $v_{L}(x) \geq \bar{v}(x)$.

To prove the reverse inequality, we take $0<\delta<1$ with $C_{\rho} \delta^{m}<\rho$ for any $\rho>0$. Then, by (2.22), we have for $|x-y|<\delta$,

$$
\begin{aligned}
\left|v_{L}(x)-v_{L}(y)\right| & \leq \sup _{c \in \mathscr{C}_{L}}\left|J_{c}(x)-J_{c}(y)\right| \leq C_{\rho} \delta^{m}+\rho\left(1+|x|^{m}+|y|^{m}\right) \\
& <\Xi_{\rho}(x):=\rho\left(2^{m}+2\right)\left(1+|x|^{m}\right) .
\end{aligned}
$$

Let $\left\{S_{i}\right\}$ be a sequence of disjoint subsets of $\mathbb{R}$ such that

$$
\operatorname{diam}\left(S_{i}\right)<\delta, \quad \bigcup_{i} S_{i}=\mathbb{R}
$$

By (2.21), for any $i$, we take $x^{(i)} \in S_{i}$ and $c^{(i)} \in \mathscr{C}_{L}$ such that

$$
J_{c^{(i)}}\left(x^{(i)}\right) \leq v_{L}\left(x^{(i)}\right)+\rho .
$$

On the other hand, by the definition of $\bar{v}(x)$, we can find $c \in \mathscr{A}_{L}$ such that

$$
\bar{v}(x)+\rho \geq E\left[\int_{0}^{\tau} e^{-\alpha t}\left\{f\left(x_{t}\right)+\left|c_{t}\right|^{m}\right\} d t+e^{-\alpha \tau} v_{L}\left(x_{\tau}\right)\right] .
$$

Define $c^{\tau} \in \mathscr{A}_{L}$ by

$$
c_{t}^{\tau}=c_{t} 1_{\{t<\tau\}}+\sum_{i} c_{t-\tau}^{(i)} 1_{x_{\tau} \in S_{i}} 1_{\{t \geq \tau\}}
$$

Then we have

$$
\begin{aligned}
& \tilde{c}_{t}^{\tau}=\sum_{i} c_{t}^{(i)} 1_{x_{\tau} \in S_{i}}, \quad \text { for } x_{\tau} \in S_{i}, \\
J_{c^{(i)}}\left(x_{\tau}\right)= & J_{c^{(i)}}\left(x_{\tau}\right)-J_{c^{(i)}}\left(x^{(i)}\right)+J_{c^{(i)}}\left(x^{(i)}\right) \\
\leq & \Xi_{\rho}\left(x_{\tau}\right)+J_{c^{(i)}}\left(x^{(i)}\right) \leq \Xi_{\rho}\left(x_{\tau}\right)+v_{L}\left(x^{(i)}\right)+\rho \\
\leq & \Xi_{\rho}\left(x_{\tau}\right)-v_{L}\left(x_{\tau}\right)+v_{L}\left(x^{(i)}\right)+v_{L}\left(x_{\tau}\right)+\rho \\
\leq & 2 \Xi_{\rho}\left(x_{\tau}\right)+v_{L}\left(x_{\tau}\right)+\rho .
\end{aligned}
$$


8 Classical solutions of linear regulator for degenerate diffusions

Using the formal Markov property, we have by Lemma 2.2,

$$
\begin{aligned}
\bar{v}(x)+\rho \geq & E\left[\int_{0}^{\tau} e^{-\alpha t}\left\{f\left(x_{t}\right)+\left|c_{t}\right|^{m}\right\} d t+e^{-\alpha \tau}\left(J_{\tau^{\tau}}\left(x_{\tau}\right)-2 \Xi_{\rho}\left(x_{\tau}\right)-\rho\right)\right] \\
= & E\left[\int_{0}^{\tau} e^{-\alpha t}\left\{f\left(x_{t}^{\tau}\right)+\left|c_{t}^{\tau}\right|^{m}\right\} d t+E\left[\int_{\tau}^{\infty} e^{-\alpha t}\left\{f\left(x_{t}^{\tau}\right)+\left|c_{t}^{\tau}\right|^{m}\right\} d t \mid \mathscr{F}_{\tau}\right]\right] \\
& -2 E\left[e^{-\alpha \tau} \Xi_{\rho}\left(x_{\tau}\right)\right]-\rho \\
= & E\left[\int_{0}^{\tau} e^{-\alpha t}\left\{f\left(x_{t}^{\tau}\right)+\left|c_{t}^{\tau}\right|^{m}\right\} d t+E\left[\int_{\tau}^{\infty} e^{-\alpha t}\left\{f\left(x_{t}^{\tau}\right)+\left|c_{t}^{\tau}\right|^{m}\right\} d t \mid \mathscr{F}_{\tau}\right]\right] \\
& -2 \rho\left(2^{m}+2\right) E\left[e^{-\alpha \tau}\left(1+\left|x_{\tau}\right|^{m}\right)\right]-\rho \\
\geq & v_{L}(x)-2 \rho\left(2^{m}+2\right)\left[\kappa_{m}\left(|x|^{m}+E\left[\int_{0}^{\tau} e^{-\alpha \tau}\left|c_{\tau}\right|^{m} d \tau\right]\right)\right]-\rho \\
\geq & v_{L}(x)-2 \rho\left(2^{m}+2\right)\left[\left(1+\kappa_{m}|x|^{m}\right)+\kappa_{m} E\left[\int_{0}^{\infty} e^{-\alpha s}\left|c_{s}\right|^{m} d s\right]\right]-\rho \\
\geq & v_{L}(x)-2 \rho\left(2^{m}+2\right)\left(1+\kappa_{m}+\frac{\kappa_{m} L^{m}}{\alpha}\right)\left(1+|x|^{m}\right)-\rho \\
= & v_{L}(x)-2\left(1+\kappa_{m}+\frac{\kappa_{m} L^{m}}{\alpha}\right) \Xi_{\rho}(x)-\rho,
\end{aligned}
$$

where $x_{t}^{\tau}$ is the response to $c_{t}^{\tau}$ with $x_{0}^{\tau}=x_{\tau}$. Letting $\rho \rightarrow 0$, we deduce $\bar{v}(x) \geq v_{L}(x)$, which completes the proof.

Theorem 2.5. Under the assumptions of Theorem 2.3, $u$ is a viscosity solution of (1.5), which satisfies (1.3) and (1.8).

Proof. We note by Lemma 2.2 that for $0 \leq \mu \leq m$,

$$
\begin{aligned}
E\left[\int_{0}^{h}\left|x_{t}\right|^{\mu} d t\right] & \leq e^{\alpha h} E\left[\int_{0}^{h} e^{-\alpha t}\left|x_{t}\right|^{\mu} d t\right] \\
& \leq e^{\alpha h} \int_{0}^{h} \kappa_{\mu}\left(|x|^{\mu}+E\left[\int_{0}^{\infty} e^{-\alpha s}\left|c_{s}\right|^{\mu} d s\right]\right) \\
& \leq e^{\alpha h} h \kappa_{\mu}\left(|x|^{\mu}+E\left[\int_{0}^{\infty} e^{-\alpha s}\left|c_{s}\right|^{\mu} d s\right]\right), \quad h>0,
\end{aligned}
$$

and by the moment inequalities for local martingales (Karatzas and Shreve [11]),

$$
\begin{aligned}
E\left[\sup _{0 \leq s \leq h}\left|x_{s}-x\right|^{\mu}\right] \leq & 3^{\mu} E\left[\left(\int_{0}^{h}\left|A x_{t}\right| d t\right)^{\mu}+\left(\int_{0}^{h}\left|c_{t}\right| d t\right)^{\mu}+\left(\sup _{0 \leq s \leq h}\left|\int_{0}^{s} \sigma x_{t} d w_{t}\right|\right)^{\mu}\right] \\
\leq & 3^{\mu}\left(|A|^{\mu} h^{\mu-1} E\left[\int_{0}^{h}\left|x_{t}\right|^{\mu} d t\right]+e^{\alpha h} h^{\mu-1} E\left[\int_{0}^{\infty} e^{-\alpha t}\left|c_{t}\right|^{\mu} d t\right]\right. \\
& \left.+C h^{\mu / 2-1} E\left[\int_{0}^{h}\left|x_{t}\right|^{\mu} d t\right]\right)
\end{aligned}
$$


for some constant $C>0$. Hence, taking $\mu=2$, we have

$$
\lim _{h \rightarrow 0} \sup _{c \in \mathscr{A}_{L}} E\left[\sup _{0 \leq s \leq h}\left|x_{s}-x\right|^{2}\right]=0
$$

Recall by (1.8) that $f$ is uniformly continuous on each compact interval. Thus we can apply a standard result of viscosity solutions (Crandall et al. [6, Theorem 3.1, page 220]) to observe that $v_{L}$ is a viscosity solution of

$$
\alpha V-\left\{\frac{1}{2} \sigma^{2} x^{2} V^{\prime \prime}+A x V^{\prime}+\min _{r \leq L}\left(|r|^{m}+r V^{\prime}\right)+f(x)\right\}=0 \quad \text { in } \mathbb{R} .
$$

Now we can define $u(x)$ by $u(x)=\lim _{L \rightarrow \infty} v_{L}(x)$ being nonincreasing. By Theorem 2.3, it is clear that $u$ fulfills (1.3) and (1.8). Thus by Dini's theorem (Apostol [1]), we can observe the locally uniform convergence and the viscosity property of $u$ (Crandall et al. [6]). The proof is complete.

\section{Classical solutions}

We studied the smoothness of the viscosity solution $u$ of (1.5). In what follows, we say that $u$ is a classical solution of (1.5) if it is twice differentiable and satisfies the equation pointwise. The value function, in general, is not smooth even for smooth systems. In order to prove the smoothness of the viscosity solution $u$ of (1.5), we used a convexity argument of $v_{L}(x), u(x)$ and the technique of viscosity solutions is used to construct solutions.

Theorem 3.1. Assume (1.3), (1.4), (1.8), and (2.8). Then

$$
u \in C^{2}(\mathbb{R} \backslash\{0\})
$$

\section{Proof}

Step 1. By the convexity of $u$, we recall a classical result of Fleming and Soner [9] to see that lebesgue measure of $\mathbb{R} \backslash \mathscr{D} \cup\{0\}=0$, where

$$
\mathscr{D}=\{x \in \mathbb{R}: u \text { is twice differentiable at } x\} .
$$

By the definition of twice differentiability, we have

$$
\left(u^{\prime}(x), u^{\prime \prime}(x)\right) \in J^{+2} u(x) \cap J^{-2} u(x) \quad \forall x \in \mathscr{D}
$$

and hence

$$
-\alpha u+\frac{1}{2} \sigma^{2} x^{2} u^{\prime \prime}+A x u^{\prime}+(1-m)\left(\frac{\left|u^{\prime}\right|}{m}\right)^{m /(m-1)}+f(x)=0 \quad \forall x \in \mathscr{D} .
$$


Let $d^{+} u(x)$ and $d^{-} u(x)$ denote the right- and the left-hand drrivatives of $u(x)$, respectively. Define $r^{ \pm}(x)$ by

$$
\begin{aligned}
& -\alpha u(x)+\frac{1}{2} \sigma^{2} x^{2} r^{ \pm}(x)+A x d^{ \pm} u(x)+(1-m)\left(\frac{\left|d^{ \pm} u(x)\right|}{m}\right)^{m /(m-1)} \\
& +f(x)=0 \quad \forall x \in(\mathbb{R} \backslash\{0\}) .
\end{aligned}
$$

Since $d^{+} u=d^{-} u=u^{\prime}$ on $\mathscr{D}$, we have $r^{+}=r^{-}=u^{\prime \prime}$ a.e. By definition, $d^{+} u(x)$ is right continuous, and so is $r^{+}(x)$. Hence it is easy to see that

$$
\begin{gathered}
u(y)-u(x)=\int_{x}^{y} d^{+} u(s) d s, \\
d^{+} u(s)-d^{+} u(x)=\int_{x}^{s} r^{+}(t) d t, \quad s>x .
\end{gathered}
$$

Thus we get

$$
\begin{aligned}
R(u ; y) & :=\frac{\left\{u(y)-u(x)-d^{+} u(x)(y-x)-1 / 2 r^{+}(x)|y-x|^{2}\right\}}{|y-x|^{2}} \\
& =\frac{\int_{x}^{y}\left(d^{+} u(s)-d^{+} u(x)-r^{+}(x)(s-x)\right) d s}{|y-x|^{2}} \\
& =\frac{\int_{x}^{y}\left\{\int_{x}^{s}\left(r^{+}(t)-r^{+}(x)\right) d t\right\} d s}{|y-x|^{2}} \longrightarrow 0 \quad \text { as } y \downarrow x .
\end{aligned}
$$

Step 2. We claim that $u(x)$ is differentiable at $x \in \mathbb{R} \backslash \mathscr{D} \cup\{0\}$. It is well known in Bardi and Capuzzo-Dolcetta [2], Clarke [5] that

$$
\partial u(x)=\left[d^{+} u(x), d^{-} u(x)\right] \quad \forall x \in(\mathbb{R} \backslash\{0\}),
$$

where $\partial u(x)$ is the generalized gradient of $u$ at $x$. Suppose $d^{+} u(x)>d^{-} u(x)$. Set

$$
\begin{gathered}
\hat{p}=\xi d^{+} u(x)+(1-\xi) d^{-} u(x), \\
\hat{r}=\xi r^{+}(x)+(1-\xi) r^{-}(x), \quad 0<\xi<1 .
\end{gathered}
$$

If

$$
\lim \inf _{y \rightarrow x} R(u ; y)<0
$$

then we can find a sequence $y_{n} \rightarrow x$ such that $\lim _{n \rightarrow \infty} R\left(u ; y_{n}\right)<0$. By (3.7), we may consider that $y_{n} \leq y_{n+1}<x$ for every $n$, taking a subsequence if necessary. Hence

$$
\lim _{n \rightarrow \infty} \frac{u\left(y_{n}\right)-u(x)-d^{+} u(x)\left(y_{n}-x\right)}{\left|y_{n}-x\right|} \leq 0,
$$


this leads to $d^{+} u(x) \leq d^{-} u(x)$, which is a contradiction. Thus we have $\left(d^{+} u(x), r^{+}(x)\right) \in$ $J^{2,-} u(x)$ and similarly, $\left(d^{-} u(x), r^{-}(x)\right) \in J^{2,-} u(x)$. By the convexity of $J^{2,-} u(x)$, we get $(\hat{p}, \hat{r}) \in J^{2,-} u(x)$. Now we note that

$$
(\hat{p})^{m}<\xi\left(d^{+} u(x)\right)^{m}+(1-\xi)\left(d^{-} u(x)\right)^{m},
$$

and hence by (3.5),

$$
-\alpha u(x)+\frac{1}{2} \sigma^{2} x^{2} \hat{r}+A x \hat{p}+\frac{(1-m)}{m^{m / m-1}}(|\partial u(x)|)^{m(2-m) /(m-1)}|\hat{p}|^{m}+f(x)>0 .
$$

On the other hand, by the definition of viscosity solution,

$$
-\alpha u(x)+\frac{1}{2} \sigma^{2} x^{2} q+A x p+(1-m)\left(\frac{|p|}{m}\right)^{m /(m-1)}+f(x) \leq 0 \quad \forall(p, q) \in J^{2,-} u(x),
$$

which is a contradiction. Therefore, we deduce that $\partial u(x)$ is a singleton, and so $u$ is differentiable at $x$ Clarke [5].

Step 3. We claim that $u^{\prime}$ is continuous on $(\mathbb{R} \backslash\{0\})$. Let $x_{n} \rightarrow x$ and $p_{n}=u^{\prime}\left(x_{n}\right) \rightarrow p$. Then by convexity, we have

$$
u(y) \geq u(x)+p(y-x) \quad \forall y .
$$

Hence we see that $p \in D^{-} u(x)$, where

$$
D^{-} u(x)=\left\{\frac{p \in \mathbb{R}: \liminf _{y \rightarrow x}\{u(y)-u(x)-p(y-x)\}}{|y-x|} \geq 0\right\} .
$$

Since $\partial u(x)=D^{-} u(x)$ and $\partial u(x)$ is a singleton, we deduce $p=u^{\prime}(x)$ by Bardi and Capuzzo-Dolcetta [2, Proposition 4.7, page 66].

Step 4. We set $w=u^{\prime}$. Since

$$
-\alpha u\left(x_{n}\right)+\frac{1}{2} \sigma^{2} x_{n}^{2} w^{\prime}\left(x_{n}\right)+A x_{n} w\left(x_{n}\right)+(1-m)\left(\frac{\left|w\left(x_{n}\right)\right|}{m}\right)^{m /(m-1)}+f\left(x_{n}\right)=0, \quad x_{n} \in \mathscr{D},
$$

the sequence $\left\{w^{\prime}\left(x_{n}\right)\right\}$ converges uniquely as $x_{n} \rightarrow x \in \mathbb{R} \backslash \mathscr{D} \cup\{0\}$, and $w$ is Lipschitz near $x$ by monotonicity. Hence, we have a well-known result in nonsmooth analysis that $\partial w(x)$ coincides with the convex hull of the set

$$
\mathbf{D}^{*} w(x)=\left\{q \in \mathbb{R}: q=\lim _{n \rightarrow \infty} w^{\prime}\left(x_{n}\right), x_{n} \in \mathscr{D} \rightarrow x\right\}
$$

Then

$$
-\alpha u(x)+\frac{1}{2} \sigma^{2} x^{2} q+A x w(x)+(1-m)\left(\frac{|w(x)|}{m}\right)^{m /(m-1)}+f(x)=0 \quad \forall q \in \partial w(x) .
$$


Hence we observe that $\partial w(x)$ is a singleton, and then $w(x)$ is differentiable at $x$. The continuity of $w^{\prime}(x)$ follows immediately. Thus we conclude that $w \in C^{1}(\mathbb{R} \backslash\{0\})$ and $(\mathbb{R} \backslash \mathscr{D} \cup\{0\})$ is empty. The proof is complete.

Theorem 3.2. Make the assumptions of Theorem 2.3. Further, assume that

$$
\begin{gathered}
f(x) \text { : convex } \\
f(x) / x^{2} \longrightarrow \hat{f} \in \mathbb{R}_{+} \text {as } x \longrightarrow 0 .
\end{gathered}
$$

Then

$$
u \in C^{1}(\mathbb{R}) \cap C^{2}(\mathbb{R} \backslash\{0\}) .
$$

In addition, if $\hat{f}=0$, then

$$
u \in C^{2}(\mathbb{R})
$$

Proof. We first observe that $v_{L}$ is a viscosity solution of the boundary value problem:

$$
\begin{aligned}
& V^{\prime \prime}+G\left(x, V, V^{\prime}\right)=0 \quad \text { in }(a, b), \\
& V(a)=v_{L}(a), \quad V(b)=v_{L}(b),
\end{aligned}
$$

for any interval $[a, b] \subset \mathbb{R} \backslash\{0\}$, where

$$
G\left(x, V, V^{\prime}\right)=\frac{2\left\{-\alpha V+A x V^{\prime}+\min _{|r| \leq L}\left(|r|^{m}+r V^{\prime}\right)+f(x)\right\}}{\sigma^{2} x^{2}}=0 .
$$

Standard elliptic regularity theory (Fleming and Soner [9, Theorem 4.1]) and the uniqueness of viscosity solutions (Crandall et al. [6]) yield that $v_{L}$ is smooth in $(a, b)$. Since $m \geq 2$, we note that

$$
\begin{aligned}
\left|\min _{|r| \leq L}\left(|r|^{m}+r v_{L}^{\prime}\right)\right| & \leq\left|\min _{r \in \mathbb{R}}\left(|r|^{m}+r v_{L}^{\prime}\right)\right|=(m-1)\left(\frac{\left|v_{L}^{\prime}\right|}{m}\right)^{m /(m-1)} \\
& \leq(m-1)\left\{\left(\frac{\left|v_{L}^{\prime}\right|}{m}\right)^{2}+1\right\} .
\end{aligned}
$$

Then by the Theorem 3.1, we have $u \in C^{2}(\mathbb{R} \backslash\{0\})$.

To prove (3.22), it suffices to show that $u$ satisfies

$$
u^{\prime}(x)=o(1) \quad \text { as } x \longrightarrow 0,
$$

since, under (3.20), $v_{L}$ and $u$ are convex by (Fleming and Soner [9, Chapter 4, Lemma 10.6]). By (3.21), there exists $\lambda>0$, for any $\varepsilon>0$ such that $f(x) \leq(\hat{f}+\varepsilon) x^{2}$ for $|x|<\lambda$, and hence, by (1.3),

$$
f(x) \leq(\hat{f}+\varepsilon) x^{2}+K\left(\frac{1}{\lambda^{m}}+1\right)|x|^{m}, \quad \forall x \in \mathbb{R} .
$$


By (2.17) and Lemma 2.2, we have $u(x) \leq E\left[\int_{0}^{\infty} e^{-\alpha t} f\left(z_{t}\right) d t\right] \leq C\left(\kappa_{2} x^{2}+\kappa_{m}|x|^{m}\right)$, and then

$$
u(x)=O\left(x^{2}\right) \quad \text { as } x \longrightarrow 0 .
$$

By convexity,

$$
u(y) \geq u(x)+u^{\prime}(x)(y-x), \quad x \neq 0 .
$$

Substituting $y=2 x$ and $y=0$, we get $u(2 x) \geq u(x)+u^{\prime}(x) x$ and $u(x)-u^{\prime}(x) x \leq u(0)=0$ by (3.29). Thus

$$
\frac{u(2 x)}{x^{2}} \geq \frac{u^{\prime}(x)}{x} \geq \frac{u(x)}{x^{2}}
$$

and then

$$
u^{\prime}(x)=O(x) \text { as } x \longrightarrow 0
$$

which implies (3.27). Finally, suppose $\hat{f}=0$. Then, by virtue of (3.28), we have $u(x)=$ $o\left(x^{2}\right)$ as $x \rightarrow 0$. Moreover, by (3.31), $u^{\prime}(x)=o(x)$ as $x \rightarrow 0$. Dividing (1.5) by $x^{2}$ and passing to the limit, we get $u^{\prime \prime}(0)=0$ which implies (3.23).

\section{Optimality}

The optimal control for the linear regulator problem is a linear function of $x(t)$. This is particularly convenient for implementation. Because of this, controls have been designed for many nonlinear problems as well as linear problems, using the solution of the linear regulator problem. We will give an optimal control of the stochastic control problem (1.1), (1.2). Let us consider the stochastic differential equation

$$
d x_{t}^{*}=\left[A x_{t}^{*}+\varphi \circ u^{\prime}\left(x_{t}^{*}\right)\right] d t+\sigma x_{t}^{*} d w_{t}, \quad x_{0}^{*}=x,
$$

where $\varphi \circ u^{\prime}\left(x_{t}^{*}\right)$ is the composite function of $\varphi$ and $u$ and $\varphi(x):=-\operatorname{sgn}(x)(|x| / m)^{1 /(m-1)}$ is the minimizer of $\min _{r \in \mathbb{R}}\left(|r|^{m}+r x\right), m \geq 0$.

The main references for this section are Ikeda and Watanabe [10], and (Karatzas and Shreve [11, page 219]). Our aim is to prove Theorem 4.1.

Theorem 4.1. Assume (1.3), (1.4), (1.8), (2.8), (3.20), and (3.21). Then the optimal control $c_{t}^{*}$ is given by

$$
c_{t}^{*}=\varphi \circ u^{\prime}\left(x_{t}^{*}\right)
$$

Proof. By (3.22), there exists a weak solution $\left(x_{t}^{*}\right)$ of (4.1) up to explosion time $\sigma=$ $\inf \left\{t:\left|x_{t}^{*}\right|=\infty\right\}$ (cf. Ikeda and Watanabe [10, Chapter 4, Theorem 2.3]). We note by (3.27) and by convexity that $x \varphi \circ u^{\prime}(x) \leq 0$ (Ikeda and Watanabe [10]). Indeed, by (3.27), $u^{\prime}(0)=0$, since $u(x)$ : convex and $u \in C^{2}(\mathbb{R})$, so $u^{\prime \prime}\left(x^{\prime}\right) \geq 0, x^{\prime} \in \mathbb{R}$. Using mean value 
theorem, $u^{\prime}(x)-u^{\prime}(0)=x u^{\prime \prime}\left(x^{\prime}\right) \geq 0$ for all $x^{\prime} \in(0, x), u^{\prime}(x) \geq 0$. Again, $u^{\prime}(0)-u^{\prime}(x)=$ $(-x) u^{\prime \prime}\left(x^{\prime}\right) \geq 0$ for all $x^{\prime} \in(x, 0), u^{\prime}(x) \leq 0$. Now $\varphi(x):=-\operatorname{sgn}(x)(|x| / m)^{1 /(m-1)}$, $\operatorname{sgn}(\varphi(x))=-\operatorname{sgn}(x), \operatorname{sgn} \varphi\left(u^{\prime}(x)\right)=-\operatorname{sgn}\left(u^{\prime}(x)\right)$, and $\operatorname{sgn} \varphi\left(u^{\prime}(x)\right)=-1$ for $x \geq 0$ and $\operatorname{sgn} \varphi\left(u^{\prime}(x)\right)=1$ for $x \leq 0$. Hence $x \varphi\left(u^{\prime}(x)\right) \leq 0$ for both $x \geq 0$ and $x \leq 0$.

Since, by Itô's formula,

$$
\begin{aligned}
d\left(x_{t}^{*}\right)^{2} & =2 x_{t}^{*} d x_{t}^{*}+\frac{1}{2} \sigma^{2}\left(x_{t}^{*}\right)^{2} 2 d t \\
& =2 x_{t}^{*}\left[\left(A x_{t}^{*}+\varphi \circ u^{\prime}\left(x_{t}^{*}\right)\right) d t+\sigma x_{t}^{*} d w_{t}\right]+\sigma^{2}\left(x_{t}^{*}\right)^{2} d t \\
d\left(z_{t}\right)^{2} & =2 z_{t} d z_{t}+\frac{1}{2} \sigma^{2}\left(z_{t}\right)^{2} 2 d t \\
& =2 z_{t}\left[A z_{t} d t+\sigma z_{t} d w_{t}\right]+\sigma^{2}\left(z_{t}\right)^{2} d t
\end{aligned}
$$

by the assumptions of the comparison theorem, we have $b_{1}(x)=A x+\varphi \circ u^{\prime}(x)+\sigma^{2}(x)^{2}$, $b_{2}(x)=A x+\sigma^{2}(x)^{2}$ then, we have $b_{1}(x)<b_{2}(x)$ and $|\sigma x-\sigma y|=|\sigma||x-y|$, where $\sigma x=$ $\rho(x)$, so $\int_{0}^{\epsilon} \rho^{-2}(x) d x=\int_{0}^{\epsilon}(\sigma x)^{-2} d x=\infty$. Thus we can see that $\left(x_{t}^{*}\right)^{2} \leq\left(z_{t}\right)^{2}$ by the comparison theorem (Ikeda and Watanabe [10]). Since the explosion time $\sigma=\inf \left\{t:\left|x_{t}^{*}\right|=\right.$ $\infty$, we have $\infty=\left(x_{\sigma}^{*}\right)^{2} \leq\left(z_{\sigma}\right)^{2}$. Hence $\sigma=\infty$. By the monotonicity of $u^{\prime}(x)$ and $\varphi(x)$, we have $\varphi \circ u^{\prime}(y)<\varphi \circ u^{\prime}(x)$ for $y<x$. Then

$$
\begin{gathered}
d y_{t}=\left[A y_{t}+\varphi \circ u^{\prime}\left(y_{t}\right)\right] d t+\sigma y_{t} d w_{t}, \quad y_{0}=x^{*}, \\
d x_{t}^{*}=\left[A x_{t}^{*}+\varphi \circ u^{\prime}\left(x_{t}^{*}\right)\right] d t+\sigma x_{t}^{*} d w_{t}, \quad x_{0}^{*}=x^{*}, \\
d\left(y_{t}-x_{t}^{*}\right)=\left[A\left(y_{t}-x_{t}^{*}\right)+\left(\varphi \circ u^{\prime}\left(y_{t}\right)-\varphi \circ u^{\prime}\left(x_{t}^{*}\right)\right)\right] d t+\sigma\left(y_{t}-x_{t}^{*}\right) d w_{t},
\end{gathered}
$$

by Itô's formula,

$$
\begin{aligned}
d\left(y_{t}-x_{t}^{*}\right)^{2}= & 2\left(y_{t}-x_{t}^{*}\right) d\left(y_{t}-x_{t}^{*}\right)+\frac{1}{2} \sigma^{2}\left(y_{t}-x_{t}^{*}\right)^{2} 2 d t \\
= & 2 A\left(y_{t}-x_{t}^{*}\right)^{2} d t+2\left(y_{t}-x_{t}^{*}\right)\left(\varphi \circ u^{\prime}\left(y_{t}\right)-\varphi \circ u^{\prime}\left(x_{t}^{*}\right)\right) d t \\
& +2 \sigma\left(y_{t}-x_{t}^{*}\right)^{2} d w_{t}+\sigma^{2}\left(y_{t}-x_{t}^{*}\right)^{2} d t \\
\leq & \left(2 A+\sigma^{2}\right)\left(y_{t}-x_{t}^{*}\right)^{2} d t+2 \sigma\left(y_{t}-x_{t}^{*}\right)^{2} d w_{t} .
\end{aligned}
$$

Hence

$$
\begin{gathered}
\left(y_{t}-x_{t}^{*}\right)^{2} \leq\left(2 A+\sigma^{2}\right) \int_{0}^{t}\left(y_{s}-x_{s}^{*}\right)^{2} d s+2 \sigma \int_{0}^{t}\left(y_{s}-x_{s}^{*}\right)^{2} d w_{s}, \\
E\left[\left(y_{t}-x_{t}^{*}\right)^{2}\right] \leq\left(2 A+\sigma^{2}\right) \int_{0}^{t} E\left[\left(y_{s}-x_{s}^{*}\right)^{2}\right] d s .
\end{gathered}
$$

Setting $\xi(t)=E\left[\left(y_{t}-x_{t}^{*}\right)^{2}\right]$, we obtain $\xi(t) \leq K \int_{0}^{t} \xi(s) d s$, for all $t \geq 0$, where $K>2 A+\sigma^{2}$. By Gronwall lemma, we have $\xi(t)=0$ a.s. for all $t \geq 0$. Therefore, $E\left[\left(y_{t}-x_{t}^{*}\right)^{2}\right]=0$ a.s. for all $t \geq 0$, from which we have $y_{t} \leq x_{t}^{*}$. So, the uniqueness of (4.1) holds. Thus we conclude that (4.1) admits a unique strong solution $\left(x_{t}^{*}\right)$ (cf. Ikeda Watanabe [10, Chapter 4, Theorem 1.1]). 
Now, we apply Itô's formula for convex functions (Karatzas and Shreve [11, 219]) to obtain

$$
\begin{aligned}
e^{-\alpha t} u\left(x_{t}^{*}\right)= & u(x)+\left.\int_{0}^{t} e^{-\alpha s}\left(-\alpha u+A x u^{\prime}+c_{s}^{*} u^{\prime}+\frac{1}{2} \sigma^{2} x^{2} u^{\prime \prime}\right)\right|_{x=x_{s}^{*}} d s \\
& +\int_{0}^{t} e^{-\alpha s} \sigma x_{s}^{*} u^{\prime} d w_{s} .
\end{aligned}
$$

By virtue of (1.5)

$$
E\left[e^{-\alpha\left(t \wedge \tau_{n}\right)} u\left(x_{t \wedge \tau_{n}}^{*}\right)\right]=u(x)-E\left[\int_{0}^{t \wedge \tau_{n}} e^{-\alpha s}\left\{f\left(x_{s}^{*}\right)+\left|c_{s}^{*}\right|^{m}\right\} d s\right]
$$

where $\left\{\tau_{n}\right\}$ is a sequence of localizing stopping times for the local martingale. Letting $n \rightarrow \infty$ and then $t \rightarrow \infty$, we get $J\left(c^{*}\right) \leq u(x)$ and $c^{*}=\left(c_{t}^{*}\right) \in \mathcal{A}$. Moreover, taking into account (2.34) with $\mu=m$, again we apply Itô's formula for convex functions (Karatzas and Shreve [11, page 219]) and we can see by the same calculation as above that

$$
\begin{aligned}
e^{-\alpha t} u\left(x_{t}\right)= & u(x)+\left.\int_{0}^{t} e^{-\alpha s}\left(-\alpha u+A x u^{\prime}+c_{s} u^{\prime}+\frac{1}{2} \sigma^{2} x^{2} u^{\prime \prime}\right)\right|_{x=x_{s}} d s \\
& +\int_{0}^{t} e^{-\alpha s} \sigma x_{s} u^{\prime}\left(x_{s}\right) d w_{s} \\
\geq & u(x)-\int_{0}^{t} e^{-\alpha s}\left\{f\left(x_{s}\right)+\left|c_{s}\right|^{m}\right\} d s+\int_{0}^{t} e^{-\alpha s} \sigma x_{s} u^{\prime}\left(x_{s}\right) d w_{s} .
\end{aligned}
$$

Again by virtue of (1.5), we have

$$
E\left[e^{-\alpha t} u\left(x_{t}\right)\right] \geq u(x)-E\left[\int_{0}^{t} e^{-\alpha s}\left\{f\left(x_{s}\right)+\left|c_{s}\right|^{m}\right\} d s\right], \quad c \in \mathscr{A} .
$$

By (1.4), we have

$$
\begin{aligned}
& E\left[\int_{0}^{\infty} e^{-\alpha t} k_{0}\left|x_{t}\right|^{m} d t\right]-E\left[\int_{0}^{\infty} e^{-\alpha t} k_{1} d t\right] \\
& \quad \leq E\left[\int_{0}^{\infty} e^{-\alpha t} f\left(x_{t}\right) d t\right] \leq E\left[\int_{0}^{\infty} e^{-\alpha t} K\left(1+\left|x_{t}\right|^{m}\right) d t\right] .
\end{aligned}
$$

Then

$$
\begin{aligned}
E\left[\int_{0}^{\infty} e^{-\alpha t}\left|x_{t}\right|^{m} d t\right] & \leq \frac{K+k_{1}}{k_{0} \alpha}+\frac{K}{k_{0}} E\left[\int_{0}^{\infty} e^{-\alpha t}\left|x_{t}\right|^{m} d t\right] \\
& \leq \frac{K+k_{1}}{k_{0} \alpha}+\frac{K \kappa_{m}}{k_{0}}\left(|x|^{m}+E\left[\int_{0}^{\infty} e^{-\alpha t}\left|c_{t}\right|^{m} d t\right]\right) \\
& \leq \frac{K+k_{1}}{k_{0} \alpha}+\frac{K \kappa_{m}}{k_{0}}|x|^{m}+\kappa_{m} \frac{K L^{m}}{k_{0} \alpha}<\infty
\end{aligned}
$$

which implies $\liminf _{t \rightarrow \infty} E\left[e^{-\alpha t}\left|x_{t}\right|^{m}\right]=0$. Therefore, we deduce $u(x) \leq J(c)$ for all $c \in \mathscr{A}$. The proof is complete. 


\section{Acknowledgment}

The author would like to express his sincere gratitude to Professor Dr. A. B. M. A. Sobhan Miah for his advice, support, and encouragement.

\section{References}

[1] T. M. Apostol, Mathematical Analysis, Addison-Wesley, Massachusetts, 1974.

[2] M. Bardi and I. Capuzzo-Dolcetta, Optimal Control and Viscosity Solutions of Hamilton-JacobiBellman Equations, Systems \& Control: Foundations \& Applications, Birkhäuser Boston, Massachusetts, 1997.

[3] R. Bellman, Dynamic Programming, Princeton Univeristy Press, New Jersey, 1957.

[4] A. Bensoussan, Stochastic Control by Functional Analysis Methods, Studies in Mathematics and Its Applications, vol. 11, North-Holland, Amsterdam, 1982.

[5] F. H. Clarke, Optimization and Nonsmooth Analysis, Canadian Mathematical Society Series of Monographs and Advanced Texts, John Wiley \& Sons, New York, 1983.

[6] M. G. Crandall, H. Ishii, and P.-L. Lions, User's guide to viscosity solutions of second order partial differential equations, American Mathematical Society. Bulletin. New Series 27 (1992), no. 1, $1-67$.

[7] M. G. Crandall and P.-L. Lions, Viscosity solutions of Hamilton-Jacobi equations, Transactions of the American Mathematical Society 277 (1983), no. 1, 1-42.

[8] G. Da Prato, Direct solution of a Riccati equation arising in stochastic control theory, Applied Mathematics and Optimization 11 (1984), no. 3, 191-208.

[9] W. H. Fleming and H. M. Soner, Controlled Markov Processes and Viscosity Solutions, Applications of Mathematics (New York), vol. 25, Springer, New York, 1993.

[10] N. Ikeda and S. Watanabe, Stochastic Differential Equations and Diffusion Processes, NorthHolland Mathematical Library, vol. 24, North-Holland, Amsterdam, 1981.

[11] I. Karatzas and S. E. Shreve, Brownian Motion and Stochastic Calculus, Graduate Texts in Mathematics, vol. 113, Springer, New York, 1988.

[12] S. Koike and H. Morimoto, Variational inequalities for leavable bounded-velocity control, Applied Mathematics and Optimization 48 (2003), no. 1, 1-20.

[13] P.-L. Lions, Optimal control of diffusion processes and Hamilton-Jacobi-Bellman equations. II. Viscosity solutions and uniqueness, Communications in Partial Differential Equations 8 (1983), no. 11, 1229-1276.

[14] J.-L. Menaldi and M. Robin, On some cheap control problems for diffusion processes, Transactions of the American Mathematical Society 278 (1983), no. 2, 771-802.

[15] M. Nisio, Lectures on Stochastic Control Theory, ISI Lecture Notes, vol. 9, MacMillan, New Delhi, 1981.

Md. Azizul Baten: Department of Statistics, Shah Jalal University of Science and Technology,

Sylhet 3114, Bangladesh

E-mail address: baten@sust.edu 


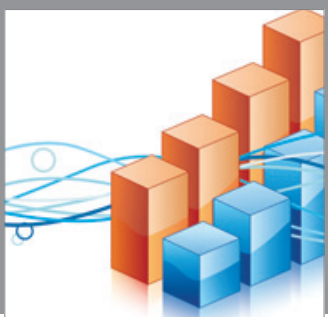

Advances in

Operations Research

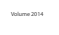

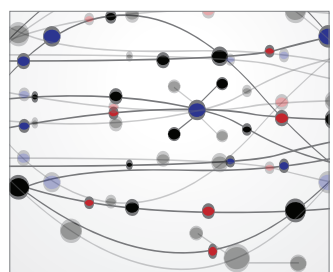

\section{The Scientific} World Journal
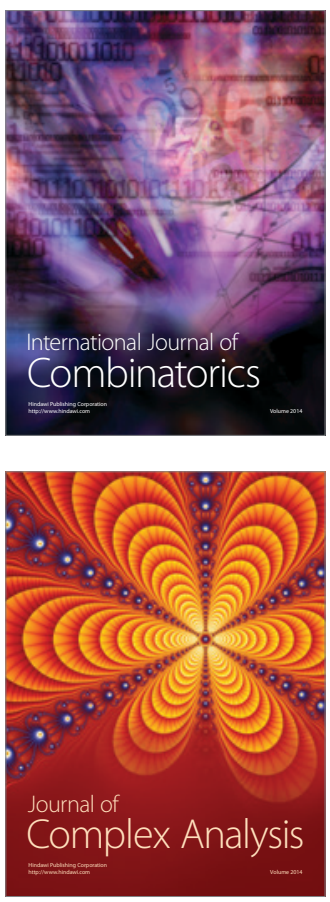

International Journal of

Mathematics and

Mathematical

Sciences
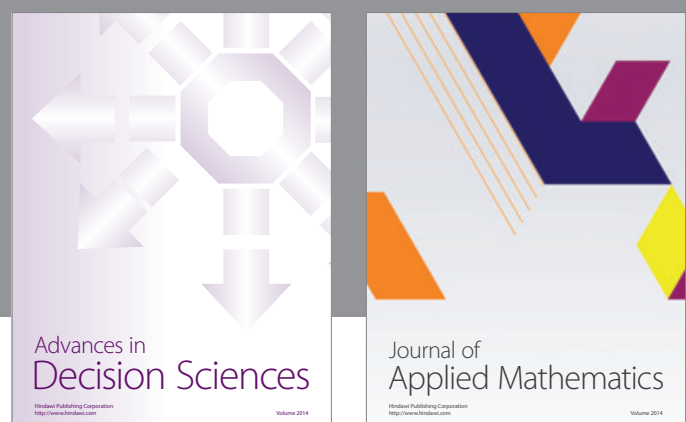

Journal of

Applied Mathematics
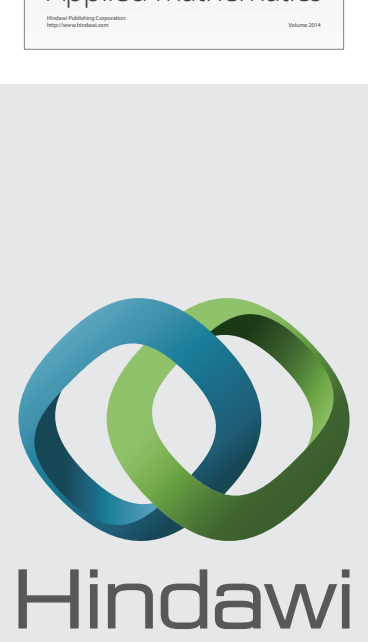

Submit your manuscripts at http://www.hindawi.com
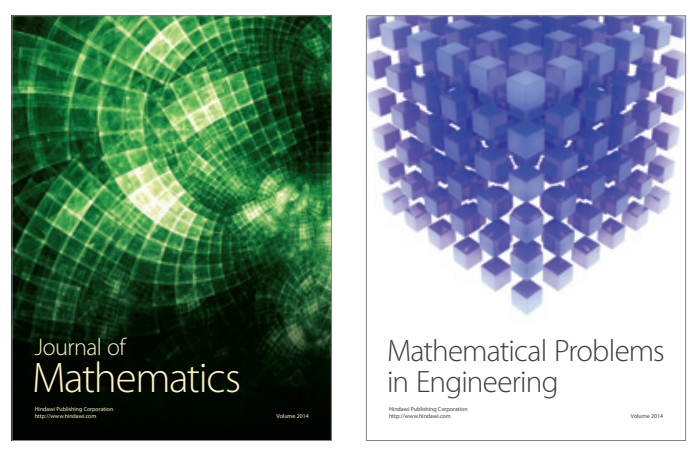

Mathematical Problems in Engineering
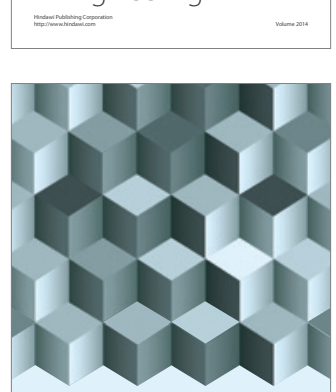

Journal of

Function Spaces
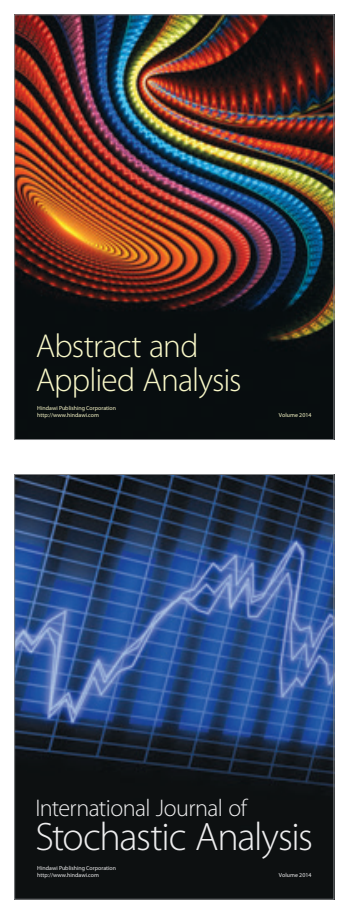

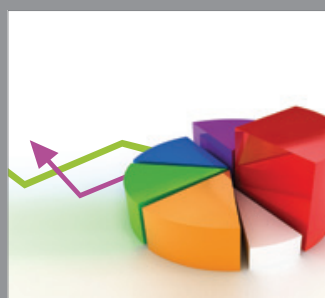

ournal of

Probability and Statistics

Promensencen
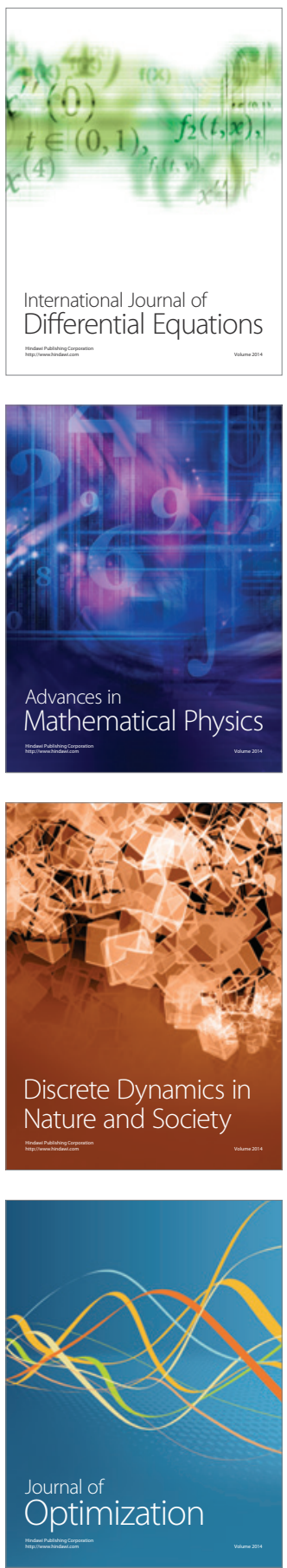